\title{
On the Buckling of Euler Graphene Beams Subject to Axial Compressive Load
}

\author{
Mohamed B. M. Elgindi ${ }^{*}$, Dongming Wei², Yeran Soukiassian1, Yu Liu ${ }^{3}$ \\ ${ }^{1}$ Department of Mathematics, Texas A \& M University-Qatar, Doha, Qatar \\ ${ }^{2}$ Department of Mathematics, University of New Orleans, Louisiana, USA \\ ${ }^{3}$ Department of Electrical Engineering, University of New Orleans, Louisiana, USA \\ Email: mohamed.elgindi@qatar.tamu.edu, dwei@uno.edu, yeran.soukiassian@qatar.tamu.edu, \\ lyu2@my.uno.edu
}

Received 11 April 2014; revised 15 May 2014; accepted 23 May 2014

Copyright (C) 2014 by authors and Scientific Research Publishing Inc.

This work is licensed under the Creative Commons Attribution International License (CC BY). http://creativecommons.org/licenses/by/4.0/

(c) (i) Open Access

\begin{abstract}
In this paper, we consider the buckling of an Euler-Bernoulli graphene beam due to an axial compressive load. We formulate the problem as a non-linear (eigenvalue) two-point boundary value problem, prove some properties of the eigenpairs and introduce a suitable numerical shooting method scheme for approximating them. We present the perturbation and the numerical approximations of the first and second buckling loads and the corresponding shapes.
\end{abstract}

\section{Keywords}

Critical Buckling Load, Graphene, Euler-Bernoulli Beam, Non-Linear Eigenvalue Problem, Shooting Method

\section{Introduction}

It is well-known from materials science, physics, and chemistry perspective, that intense interest in graphene material is developing at an accelerating pace and has recently generated numerous publications and research. Applications and the potential for graphene made structures are abundant. For instance, numerous engineering nanoscale devices that use graphene as basic components, like nanoscale resonators, switches, and valves, are being developed by many industries. Understanding the response of individual graphene structure elements to applied loads is crucially important (see [1]-[9] and the reference there in for a comprehensive list of applications).

The Euler buckling load of simply supported straight elastics beam subject to an end axial compressive load

*First author.

How to cite this paper: Elgindi, M.B.M., et al. (2014) On the Buckling of Euler Graphene Beams Subject to Axial Compressive Load. World Journal of Engineering and Technology, 2, 149-158. http://dx.doi.org/10.4236/wjet.2014.22016 
can be modeled by the equation:

$$
E I v^{\prime \prime \prime}+P v^{\prime \prime}=0, \quad 0<x<L
$$

with boundary conditions

$$
v(0)=v(L)=v^{\prime \prime}(0)=v^{\prime \prime}(L)=0
$$

where $L$ is the length of the beam, $E$ the Young's modulus, and $I$ the area moment of inertia.

Integrating (1.1) twice gives:

$$
E I v^{\prime \prime}+P v=c_{1}+c_{2} x
$$

and applying boundary conditions (1.2), we get: $c_{1}=c_{2}=0$.

The boundary value problem (1.1), (1.2), then reduces to:

$$
\left\{\begin{array}{l}
E I v^{\prime \prime}+P v=0 \\
v(0)=v(L)
\end{array}\right.
$$

The general solution of Equation (1.3) is

$$
v(x)=A \cos \left(\sqrt{\frac{P}{E I}} x\right)+B \sin \left(\sqrt{\frac{P}{E I}} x\right),
$$

where $A$ and $B$ are arbitrary constants to be determined so that the boundary conditions are satisfied. This gives a sequence of non-zero (Eigenvalues/functions) solutions:

$$
P_{k}=E I\left(\frac{k \pi}{L}\right)^{2}, \quad v_{k}(x)=\sin \left(\frac{k \pi x}{L}\right), k=1,2,3, \cdots
$$

Furthermore, each eigenvalue of (1.3) is positive and simple, and satisfy $\lim _{n \rightarrow \infty} P_{k}=\infty$. The first eigenfunction $v_{1}(x)$ is called the first buckling mode and the load corresponding to the first eigenvalue is called the critical buckling load. This buckling load is known as Euler's Buckling load and is widely used in engineering applications.

The buckling analysis presented above is based on the Hooke's law, relating the stress by: $\sigma_{x}$ and strain $\epsilon_{x}$ by: $\sigma_{x}=E \epsilon_{x}$, and the assumption that during the deformation, the cross-sections of the beam column remains perpendicular to its center line. These classical results are generalized for material that follow the Hollomon's law $\sigma_{x}=K\left|\epsilon_{x}\right|^{n-1} \epsilon_{x}, 0<n \leq 1$, with $n=1$ corresponding to Hook's law. In this case the boundary value problem (1.1), (1.2) is replaced by:

$$
\left\{\begin{array}{c}
\left(\left|v^{\prime \prime}\right|^{n-1} v^{\prime \prime}\right)+\lambda v^{\prime \prime}=0, \quad 0<x<L \\
v(0)=v(L)=v^{\prime \prime}(0)=v^{\prime \prime}(L)=0
\end{array}\right.
$$

The first eigenvalue of (1.5) is found in [10], and is given by:

$$
\lambda_{1}=\frac{2 n}{n+1}\left(\frac{\pi_{2,1+1 / n}}{L^{n}}\right)^{2}
$$

which leads to the critical load:

$$
P_{c r}=\frac{2 n\left(\pi_{2,1+1 / n}\right)^{2}}{n+1} K I_{n}
$$

where $K$ is a material constant,

$$
I_{n}=\iint_{A}|y|^{1+n} \mathrm{~d} y \mathrm{~d} z
$$

is the generalized area moment of inertia, and

$$
\pi_{2,1+1 / n}=2 \int_{0}^{\pi / 2}(\cos \theta)^{(n-1) /(n+1)} \mathrm{d} \theta
$$


The first eigenfunction is defined in terms the of the generalized sine function $\sin _{2,1+1 / n}(x)$, using the notation of the two parameter sine function developed in [11]. It is well known that the eigenvalues of (1.5) are all positive, simple and form an increasing sequence to infinity. These facts follow from some well-known results about the eigenvalue problem corresponding to the p-Laplace operator [12].

The purpose of this paper is to consider the buckling of an Euler-Bernoulli beam made of graphene material acted upon by an axial compressive load, formulate the equilibrium equations and introduce a suitable numerical technique for solving them. We are also interested in examining the dependence of the critical buckling load on the graphene's quadratic term parameter.

Graphene materialis are shown to be modeled by the following quadratic stress-strain constitutive law (see [2] and [7]):

$$
\sigma_{x}=E \varepsilon_{x}+D\left|\varepsilon_{x}\right| \varepsilon_{x}
$$

where $D$ is related to the Young's modulus by the relation:

$$
D=-\frac{E^{2}}{4 \sigma_{\max }}
$$

where $\sigma_{\max }$ is the material's ultimate maximal axial stress. We notice that for small strain, the elastic stress $E \sigma_{x}$ dominates in (1.6), while the plastic stress, $D\left|\varepsilon_{x}\right| \varepsilon_{x}$, becomes prominent with large deformation. The ratio:

$$
\left|\frac{D}{E}\right|=\frac{E}{4\left|\sigma_{\max }\right|} \equiv \delta
$$

is known as the elastoplastic parameter. When this parameter is small the material's ultimate maximal shear stress $\sigma_{\max }$ is very large, and the elastic behavior dominates.

The eigenvalue problem corresponding to (1.5) for a beam made of graphene is given by:

$$
\left\{\begin{array}{l}
\frac{\mathrm{d}^{4} w}{\mathrm{~d} z^{4}}-\alpha \frac{\mathrm{d}^{2}}{\mathrm{dz} z^{2}}\left(\left|w^{\prime \prime}\right| w^{\prime \prime}\right)^{\prime \prime}+\lambda w^{\prime \prime}=0,0<z<1 \\
w(0)=w(1)=w^{\prime \prime}(0)=w^{\prime \prime}(1)=0
\end{array}\right.
$$

where we used the following non-dimensional variables and parameters:

and

$$
z=x L^{-1}, w=v L^{-1}, \alpha=\frac{|D| I_{2}}{E I L}, \lambda=\frac{P L^{2}}{E I},
$$

$$
I=\iint_{A} y^{2} \mathrm{~d} y \mathrm{~d} z, \quad I_{2}=\iint_{A}|y|^{3} \mathrm{~d} y \mathrm{~d} z
$$

Note that in the above formulas the $z$-axis being in the off-plane direction and $A$ is the cross sectional area.

In [13], it was shown that the eigenvalues of (1.7) form a sequence of positive real numbers which tends to infinity and that each eigenvalue is simple. In this paper we are concerned with perturbation and numerical approximations of the eigenvalues and the eigen modes and in their dependence on the parameter $\alpha$.

In Section 2, we provide an asymptotic expansion of the first eigenpairs of (1.7) in terms of a perturbation parameter. In Section 3 we verify some properties of the solutions of (1.7). In Section 4 we present the numerical approximation of the first two eigen pairs for the grapheme Euler beam.

\section{Buckling Analysis of the Graphene Beam}

Integrating (1.7) twice, and applying the boundary conditions we obtain the nonlinear eigenvalue problem:

$$
\left\{\begin{array}{l}
w^{\prime \prime}-\alpha\left|w^{\prime \prime}\right| w^{\prime \prime}+\lambda w=0 \\
w(0)=w(1)=0
\end{array}\right.
$$

When $\alpha=0$, (2.1) is reduced to the eigenvalue problem for the Euler elastic beam:

$$
\left\{\begin{array}{l}
w^{\prime \prime}+\lambda w=0 \\
w(0)=w(1)=0
\end{array}\right.
$$


whose first eigenpairs are given by:

$$
\lambda_{1}=\pi^{2}, \quad w_{1}=\sin (\pi x)
$$

We consider the expansions of the solutions of (2.1), $w$ and $\lambda$, in terms of $\alpha$ in the forms:

$$
\left\{\begin{array}{l}
w(\alpha)=w_{1}+w_{2} \alpha+O\left(\alpha^{2}\right) \\
\lambda(\alpha)=\lambda_{1}+\lambda_{2} \alpha+O\left(\alpha^{2}\right)
\end{array}\right.
$$

We substitute (2.4) in (2.1) and compare the powers of $\alpha$. The zero's order BVP is (2.2) whose solution is given by (2.3). The first order equation then reads:

$$
\left\{\begin{array}{l}
\frac{\mathrm{d}^{2} w_{2}}{\mathrm{~d} x^{2}}+\lambda_{1} w_{2}=-w_{1}^{\prime \prime 2}-\lambda_{2} w_{1} \\
w_{2}(0)=w_{2}(1)=0
\end{array}\right.
$$

whose solvability condition gives

$$
\lambda_{2}=-\int_{0}^{1} w_{1}^{\prime \prime 2} w_{1} \mathrm{~d} x / \int_{0}^{1} w_{1}^{2} \mathrm{~d} x=-\frac{8}{3} \pi^{3}
$$

This way we obtain an asymptotic expansion:

$$
\left\{\begin{array}{l}
w(x)=w_{1}(x)+w_{2}(x) \alpha+O\left(\alpha^{2}\right) \\
\lambda=\lambda_{1}+\lambda_{2} \alpha+O\left(\alpha^{2}\right)
\end{array}\right.
$$

valid for small enough $\alpha$, where $w_{2}$ is the unique solution of the boundary value problem (2.5) given by:

$$
w_{2}=\frac{2 \pi^{2}}{3} \cos (\pi x)-\frac{\pi^{2}}{6} \cos (2 \pi x)-\frac{4 \pi^{2}}{3} x \cos (\pi x)-\frac{\pi^{2}}{2}
$$

\section{Properties of the Eigenvalues and Eigenfunctions}

In this section we examine some properties of the solutions of the eigenvalue problem:

$$
\left\{\begin{array}{l}
w^{\prime \prime \prime \prime}-\alpha\left(w^{\prime \prime}|w|^{\prime \prime}\right)^{\prime \prime}+\lambda w^{\prime \prime}=0 \\
w(0)=w(1)=w^{\prime \prime}(0)=w^{\prime \prime}(1)=0
\end{array}\right.
$$

We observe that if $(\lambda, w)$ is an eigenpair of (3.1) for some $\alpha>0$, then $(\lambda,-w)$ is also an eigenpair of (3.1) corresponding to the same $\alpha$, while $(\lambda, \beta w)$ is an eigenpair of (3.1) corresponding to $\beta \alpha$, for $\beta \neq 0$. In order to prove some properties that $(\lambda, w)$ satisfy, we write (3.1) in the equivalent form:

$$
\left\{\begin{array}{l}
u^{\prime \prime}-\alpha(u|u|)^{\prime \prime}+\lambda u=0, \\
u(0)=u(1)=0,
\end{array}\right.
$$

where $u=w^{\prime \prime}$. We prove the following results.

Theorem 3.1: For every $\alpha>0$, any eigenpair of $(\lambda, u)$ of (3.2) satisfies $\lambda>0$.

Proof: Multiplying both sides of (3.2) by $(u-\alpha|u| u)^{\prime}$ and integrating, we get:

$$
\frac{1}{2}\left[(u-\alpha|u| u)^{\prime}\right]^{2}+\lambda\left[\frac{1}{2} u^{2}-\frac{2 \alpha}{3}|u|^{3}\right]=A
$$

where $A$ is a constant of integration. We consider two cases:

Case 1: Assume there is $c \in(0,1)$ such that $w(c)=\frac{1}{2 \alpha}$. In this case evaluating (3.3) at $x=0$ and $x=c$ gives: 


$$
\frac{1}{2}\left(u^{\prime}(0)\right)^{2}=\frac{1}{24 \alpha^{2}} \lambda,
$$

which implies that $\lambda \geq 0$. However, $\lambda=0$ implies that $u=0$. Therefore, $\lambda>0$.

Case 2: Assume that $|u(x)|<\frac{1}{2 \alpha}$ for $x \in[0,1]$. Multiplying (3.2) by $u^{\prime}$ and integrating by parts, we get:

$$
\int_{0}^{1} u^{\prime 2}(1-2 \alpha|u|) \mathrm{d} x=\lambda \int_{0}^{1} u^{2} \mathrm{~d} x
$$

which in turn implies that $\lambda>0$.

Theorem 3.2: For any $\alpha>0$, any eigenpair $(\lambda, u)$ of (3.2) must satisfy $|u|<\frac{1}{2 \alpha}$ on $[0,1]$.

Proof: Assume that the conclusion of the theorem is false. Then, without loss of generality we can assume that $u$ has a local maximum at $c \in(0,1)$ with $u(c) \geq \frac{1}{2 \alpha}$. Then $u^{\prime}(0)=0$, and $u^{\prime \prime}(c) \leq 0$. However, (3.2) gives

$$
u^{\prime \prime}(1-2 \alpha|u|)-2 \alpha \frac{u u^{\prime 2}}{|u|}+\lambda u=0,
$$

which upon evaluation at $x=c$ gives:

$$
u^{\prime \prime}(c)(1-2 \alpha|u(c)|)+\lambda u(c)=0
$$

which is a contradiction.

Using Theorem 3.2, we will obtain an equivalent boundary value problem to (3.1) which we will use in the next section to construct the numerical solution. Integrating the differential equation in (3.1) two times, and using the boundary conditions, we obtain the boundary value problem:

$$
\left\{\begin{array}{l}
w^{\prime \prime}-\alpha w^{\prime \prime}\left|w^{\prime \prime}\right|+\lambda w=0, \\
w(0)=w(1)=0,
\end{array}\right.
$$

which we can write as:

$$
\left\{\begin{array}{l}
\phi\left(w^{\prime \prime}\right)+\lambda w=0, \\
w(0)=w(1)=0,
\end{array}\right.
$$

where $\phi(x)=x-\alpha|x| x$. Since $\phi(x)$ is invertible for $|x| \leq \frac{1}{2 \alpha}$, and since any eigenpair $(\lambda, w)$ of (3.1) satisfies $\left|w^{\prime \prime}\right| \leq \frac{1}{2 \alpha}, \quad \lambda>0$, it follows that the eigenvalue problems, (3.1) and (3.4) are equivalent. Furthermore, by differentiating (3.4) we obtain:

$$
\left\{\begin{array}{l}
w^{\prime \prime \prime}=\frac{-\lambda w^{\prime}}{1-2 \alpha\left|w^{\prime \prime}\right|} \\
w(0)=w(1)=w^{\prime \prime}(0)=w^{\prime \prime}(1)=0
\end{array}\right.
$$

Once again, using Theorem (3.2), it follows that the eigenvalue problem (3.6) is equivalent to (3.4) (and hence to (3.1)). In the next section we use the boundary value problem (3.6) to develop the numerical solutions of (3.1) that satisfy the additional constraint:

$$
w^{\prime}(0)=1,
$$

which in turn ensures that $w(x)$ is non-zero. 


\section{The Numerical Solution}

In this section we use the eigenvalue problem

$$
\left\{\begin{array}{l}
w^{\prime \prime \prime}=\frac{-\lambda w^{\prime}}{1-2 \alpha\left|w^{\prime \prime}\right|}, \\
w^{\prime}(0)=1, w(0)=w(1)=w^{\prime \prime}(0)=w^{\prime \prime}(1)=0,
\end{array}\right.
$$

to construct the eigenpairs $(\lambda(\alpha), w(\alpha))$ of (3.1) as functions of the elastoplastic parameter $\alpha$. We do this by treating $\lambda$ and $\alpha$ as unknown parameters to be determined along with the solution $w(x)$. Therefore, for a given $\lambda$ and $\alpha$ we integrate the initial value problem:

$$
\left\{\begin{array}{l}
w^{\prime \prime \prime}=\frac{-\lambda w^{\prime}}{1-2 \alpha\left|w^{\prime \prime}\right|} \\
w^{\prime}(0)=1, w(0)=w^{\prime \prime}(0)=0
\end{array}\right.
$$

for $w(x)$, and seek the values of $\lambda$ and $\alpha$ for which

$$
w(1)=0 \text {, and, } w^{\prime \prime}(1)=0
$$

We use Newton's method to determine $\lambda$ and $\alpha$ which satisfy the algebraic system (4.3).

Remarks:

For the first eigenvalue, $\lambda_{1}(\alpha)$, we may assume that the corresponding eigenvector $w(x)$ satisfies $w^{\prime \prime}(x) \geq 0$. This allows us to remove the absolute value from the boundary value problem (4.2).

For the second eigenvalue, $\lambda_{2}(\alpha)$, we may assume that the corresponding eigenfunction $w(x)$ satisfies $w^{\prime \prime}(x) \geq 0$ for $0 \leq x \leq \frac{1}{2}, w\left(\frac{1}{2}\right)=0, w^{\prime \prime}\left(\frac{1}{2}\right)=0$, and $w(x)$ is symmetric about the vertical line $x=\frac{1}{2}$. Therefore, in solving for $\lambda_{2}(\alpha)$ and its corresponding eigenvector we will solve the systems (4.2), (4.3) over $\left[0, \frac{1}{2}\right]$ instead of $[0,1]$ and use the symmetry property of $w(x)$ to obain $w(x)$ on $[0,1]$.

\section{Numerical Results}

Our numerical results for $\lambda_{1}(\alpha)$ are shown in Figures 1-3, while the results for $\lambda_{2}(\alpha)$ are shown in Figures 4-6.

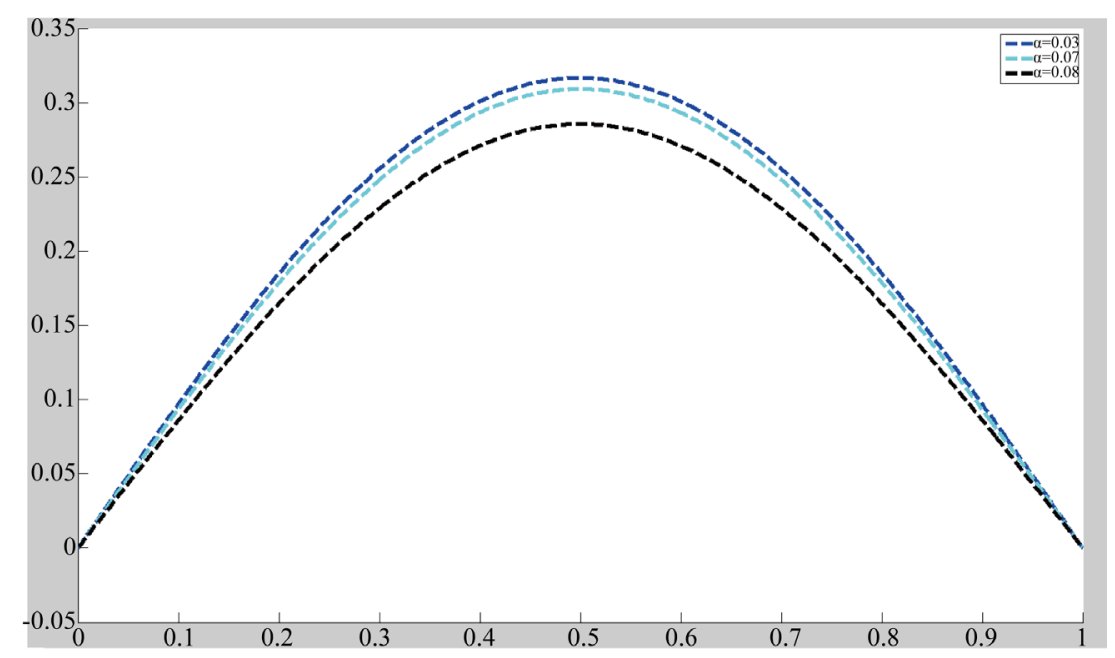

Figure 1. The first eigenfunction $w(x)$ for different values of $\alpha$. 


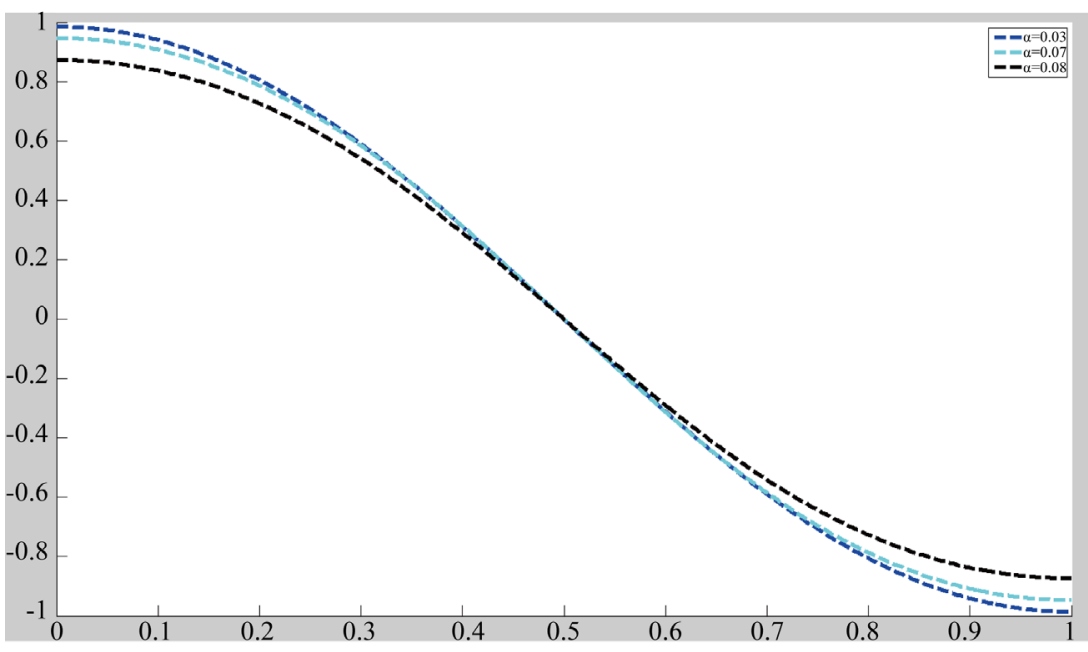

Figure 2. $w^{\prime}(x)$ for the first eigenfunction for different values of $\alpha$.

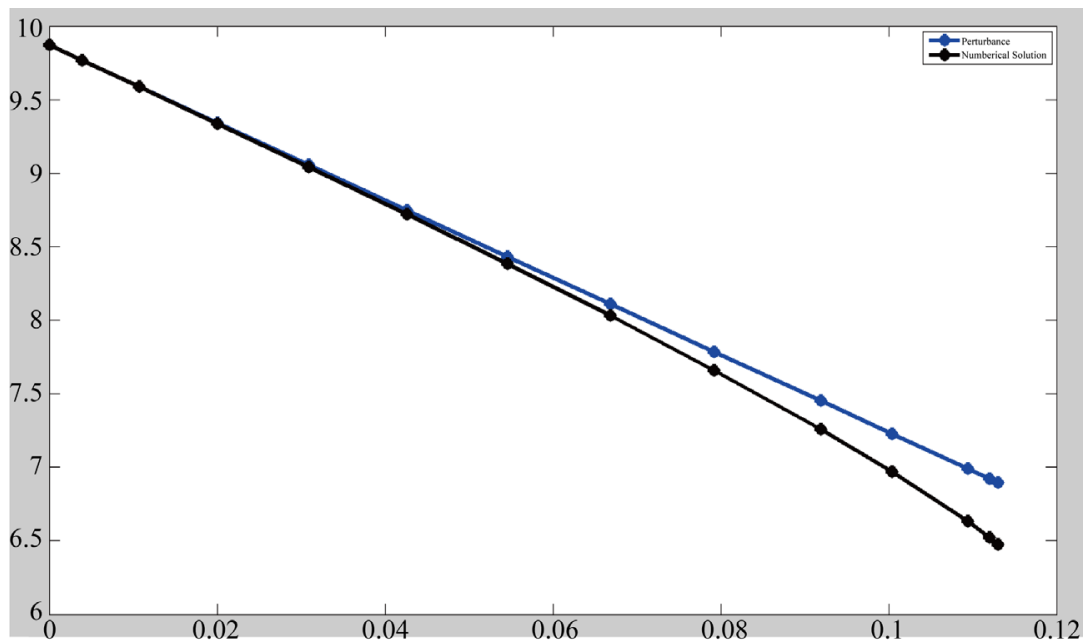

Figure 3. The first eigenvalue for different values of $\alpha$.

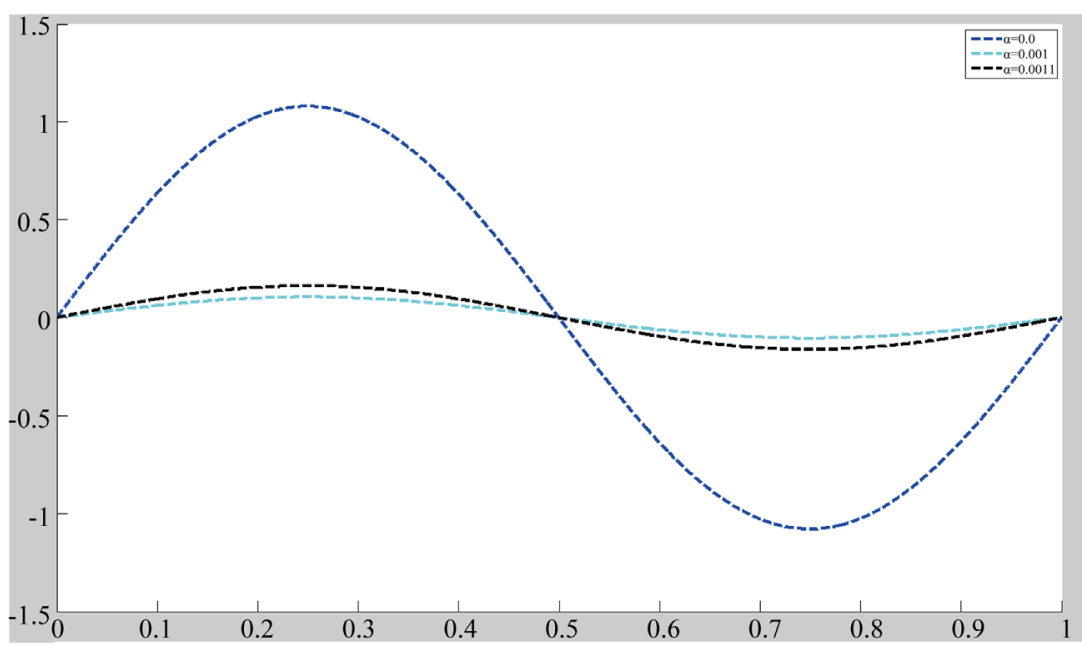

Figure 4. The second eigenfunction $w(x)$ for different values of $\alpha$. 


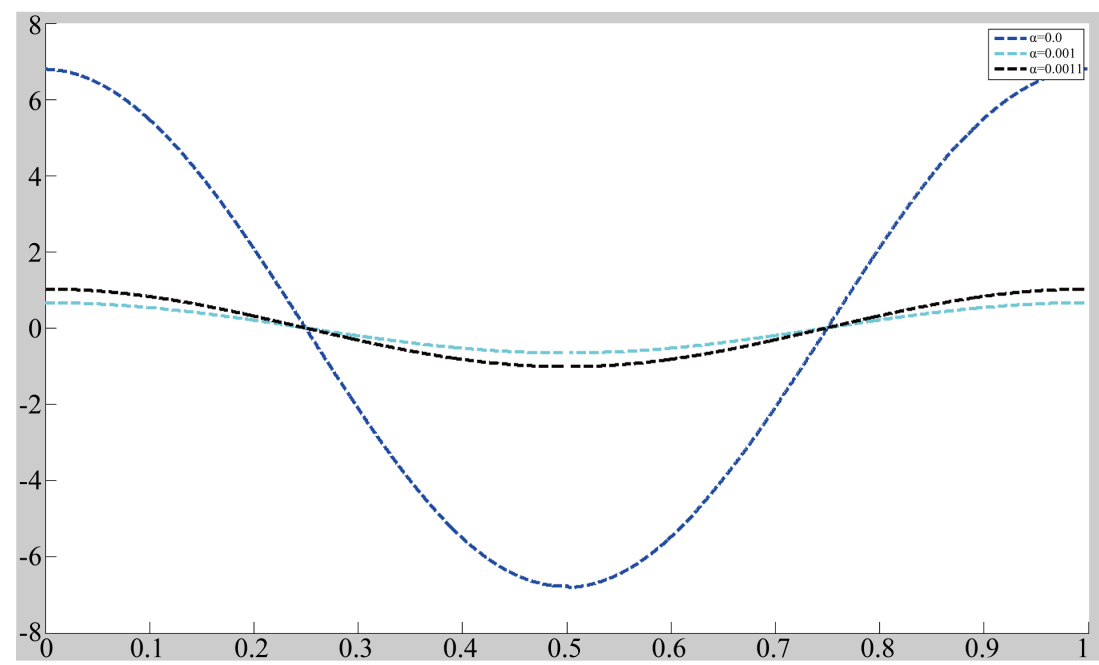

Figure 5. $w^{\prime}(x)$ for the second eigenfunction for different values of $\alpha$.

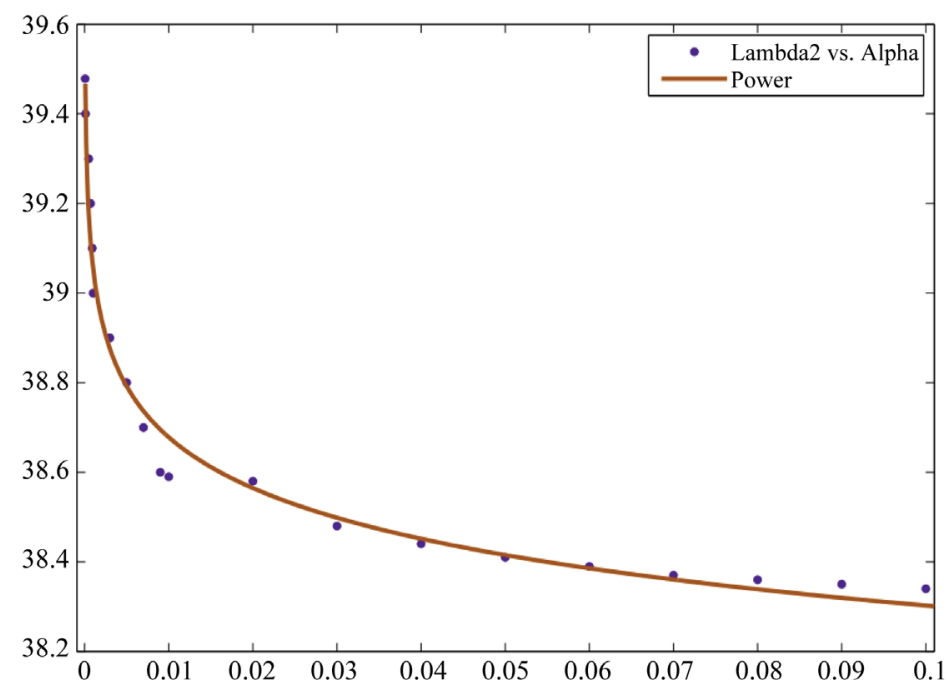

Figure 6. The second eigenvalue for different values of $\alpha$.

Below we present the numerical results for an example where the parameters have the units used in practice, [14]. We consider a beam of rectangular cross section with the dimensions: height $h=62.4 \mathrm{~nm}$, width $b=62.4$ nm, and length $L=1000 \mathrm{~nm}$ (the corresponding $\alpha=\frac{|D| I_{2}}{E I L}=0.045$ ). We take $E=1000 \mathrm{Gpa}, D=-1923 \mathrm{Gpa}$. The moment inertial $I_{1}$ and $I_{2}$ are computed by $I_{n}=\left(\frac{2 b}{n+2}\right)\left(\frac{h}{2}\right)^{n+2}, n=1,2$, [10]. The first eigenvalue is given by $\lambda=6.6049$. The corresponding criticial buckling load is $P=8.9966 \times 10^{-6} \mathrm{Gpa}$.

In this case the perturbation analysis of Section 2 , gives $\lambda \approx \lambda_{1}+\lambda_{2} \alpha=6.1489$ and $w(x) \approx w_{1}(x)+w_{2}(x) \alpha$. In Figures 7-9. we compare the numerical results to the results obtained from the perturbation solution.

\section{Conclusion}

We considered the buckling of an Euler-Bernoulli beam made of graphene. We used the properties of the buckling functions proved in Section 3 to develop equivalent boundary value problems which we treat numerically using the shooting method. We presented the numerical results for the first two eigenpairs and compared them 


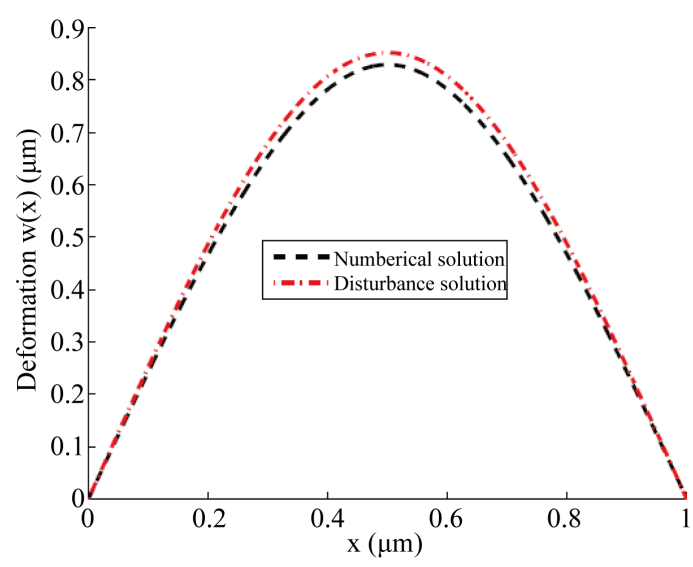

Figure 7. The first eigenfunction $w(x)$ for $\alpha=0.045$.

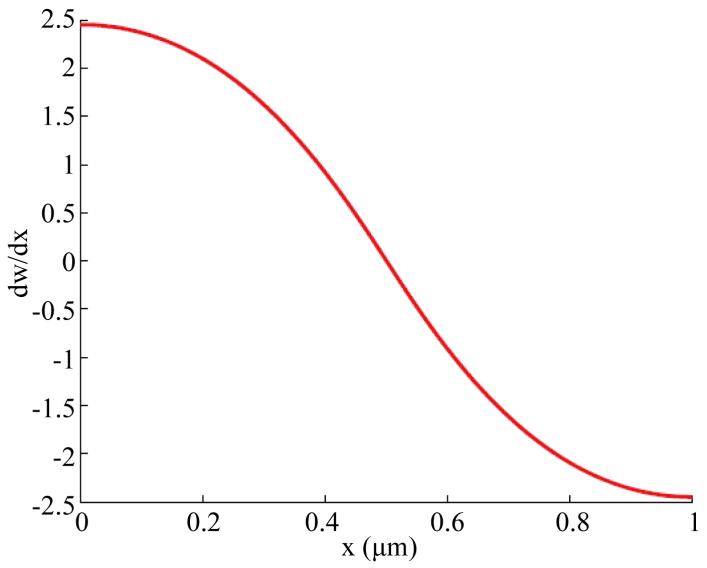

Figure 8. $w^{\prime}(x)$ for $\alpha=0.045$.

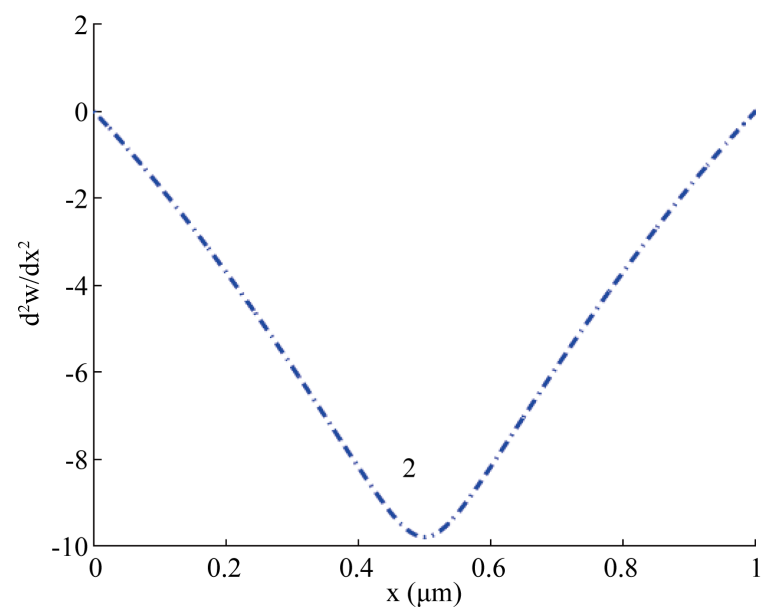

Figure 9. $w^{\prime \prime}(x)$ for $\alpha=0.045$.

to the solutions obtained by perturbation methods. We also presented the numerical results for an example using the units used in practice. Our numercial results indicate that the critical buckling load for a graphene Euler-Bernoulli beam is decreased as the graphene's qaudratic parameter is increased. 


\section{Acknowlegements}

The research in this paper is a part of a research project funded by the Research office, Texas A \& M University at Qatar.

\section{References}

[1] Zhu, Y., Murali, S., Cai, W., Li, X., Suk, J.W., Potts, J.R. and Ruoff, R.S. (2010) Graphene and Graphene Oxide: Synthesis, Properties, and Applications. Advanced Materials, 22, 3906-3924. http://dx.doi.org/10.1002/adma.201001068

[2] Galiotis, C. (2011) Mechanical Properties of Graphene. Graphene 2020/Brussels.

[3] Kim, M.T. (1996) Influence of Substrates on the Elastic Reaction of Films for the Microindentation Tests. Thin Solid Films, 283, 12-16. http://dx.doi.org/10.1016/0040-6090(95)08498-3

[4] Lee, C., Wei, X., Kysar, J.W. and Hone, J. (2008) Measurement of the Elastic Properties and Intrinsic Strength of Monolayer Graphene. Science, 321, 385-388. http://dx.doi.org/10.1126/science.1157996

[5] Lee, C., Wei, X., Li, Q., Carpick, R., Kysar, J.W. and Hone, J. (2009) Elastic and Frictional Properties of Graphene. Physica Status Solidi (B), 246, 2562-2567. http://dx.doi.org/10.1002/pssb.200982329

[6] Sansoz, F. and Gang, T. (2010) A Force-Matching Method for Quantitative Hardness Measurements by Atomic Force Microscopy with Diamond-Tipped Sapphire Cantilevers. Ultramicroscopy, 111, 11-19. http://dx.doi.org/10.1016/j.ultramic.2010.09.012

[7] Malina, E.W. (2011) Mechanical Behavior of Atomically Thin Graphene Sheets Using Atomic Force Microscopy Nanoindentation. Master's Thesis, University of Vermont, Vermont.

[8] Elgindi, M.B.M. and Wei, D.M. (2012) On the Global Solvability of a Class of Fourth-Order Nonlinear Boundary Value Problems. Applied Mathematical Sciences, 6, 5981-5992.

[9] Li, P., You, Z. and Cui, T.H. (2012) Graphene Catilever Beams for Nano Switches. Applied Physics Letters, 101, 093111. http://dx.doi.org/10.1063/1.4738891

[10] Wei, D., Sarria, A. and Elgindi, M.B.M. (2012) Critical Buckling Loads of the Perfect Hollomon's Power-law Columns, Mechanics Research Communications. 1-12.

[11] Barkat, A.B. and Matti, V. (2012) On Generalized Trigonometric Functions with Two Parameters.

[12] Drï£¡bek, P. and Manï£jsevich, R. (1999) On the Closed Solution to Some pLaplacian Nonho-Mogeneous Eigenvalue Problems. Differential Integral Equations, 12, 773-788.

[13] Elgindi, M.B.M., Wei, D. and Elgindi, T.M. (in Press) On the Solvability of an Euler Graphene Beam Subject to Axial Compressive Load.

[14] Malina, E.W. (2011) Mechanical Behavior of Atomically Thin Graphene Sheets Using Atomic Force Microscopy Nanoin-dentation. Master Thesis, University of Vermont, Vermont. 